\title{
Sincronização em Metapopulações com Hierarquia na Dinâmica Local
}

F.T. GIORDANI ${ }^{1}$, J.A.L. da SILVA ${ }^{2}$, Programa de Pós-Graduação em Matemática Aplicada, Universidade Federal do Rio Grande do Sul, UFRGS, Av. Bento Gonçalves, 9500, 91.509-900 Porto Alegre, RS, Brasil.

Resumo. Neste trabalho investigamos a possibilidade de órbitas caóticas oscilarem de forma sincronizada em modelos metapopulacionais de $k$ espécies submetidos a migração dependente da densidade. Consideramos a dinâmica local de forma hierárquica e obtemos um critério para a estabilidade do estado sincronizado de órbitas caóticas sincronizadas.

\section{Introdução}

Vários trabalhos têm dado significativa importância ao estudo de sincronização em sistemas caóticos acoplados (ver [7], [8], [19]). Este fenômeno de sincronização tem sido investigado no contexto de biologia, física, química, teoria de comunicação. Em particular, em biologia, destacam-se os modelos metapopulacionais espacialmente explícitos.

No contexto de redes de populações acopladas, a sincronização é um importante conceito, pois se a dinâmica global do sistema não está em sincronia, a população local pode ser recolonizada pelos indivíduos (migrantes) das populações vizinhas ("rescue effect"). Isso favorece a persistência da população (ver Allen et al. [1] e Heino et al. [13]). Em Gonzalez et al. [11], por exemplo, o efeito resgate ("rescue effect") é caracterizado como um importante mecanismo para permitir a persistência da espécie.

A relação entre a taxa de migração e a possibilidade de órbitas caóticas oscilarem de forma sincronizada pode ser vista também em Solé \& Gamarra [27]; uma condição simples para a estabilidade do estado síncrono envolvendo apenas a fração migratória e o expoente de Liapunov da dinâmica local foi obtida, considerando-se uma rede de 2 sítios. Em Silva et al. [24] um resultado mais geral é estabelecido, ou seja, considerando-se uma metapopulação de $n$ sítios em forma de anel. Nesse caso, a condição de estabilidade do sistema está associada ao tamanho da metapopulação, reforçando assim os resultados numéricos de Hassell et al. [12] e Comins [4] que relacionam a persistência da metapopulação com o seu tamanho.

A importância do mecanismo de migração dependente da densidade na sincronização de populações acopladas espacialmente foi enfatizado por Ims \& Andreassen

\footnotetext{
${ }^{1}$ ftgiordani@gmail.com, Bolsista do CNPq - Brasil

2jaqx@mat.ufrgs.br
} 
[14]. Este tópico foi retomado por Silva \& Giordani [25], considerando-se um tipo específico de migração dependente da densidade; nesse caso, resultados analíticos e numéricos indicam que a migração dependente da densidade induz menos sincronização na dinâmica global do sistema. Uma relação entre o grau de coerência das oscilações em cada sítio e o risco da extinção da metapopulação com dispersão independente da densidade foi estabelecida por Earn et al. [9]; um critério simples de estabilidade envolvendo o número de Liapunov da dinâmica local, a freqüência da migração e a matriz de configuração da rede foi obtido. Estes resultados (descritos em [9] e [25]) foram estendidos por Silva et al. [22] para um processo mais geral de migração dependente da densidade.

Neste trabalho analisaremos a relação entre migração dependente da densidade e sincronismo de órbitas caóticas para um modelo metapopulacional de $k$ espécies. Inicialmente reduzimos a dificuldade da análise da estabilidade local do modelo via linearização do sistema original. Dessa forma, estendemos o trabalho de Jansen \& Lloyd [15] que obtiveram resultado similar para um modelo "multi-patch" com migração constante. Consideramos a dinâmica local de forma hierárquica e estabelecemos um critério para a estabilidade local do estado sincronizado, estendendo assim os resultados de [9], [22] e [25].

\section{O Modelo}

Consideramos uma coleção de $n$ sítios enumerados por $1,2, \ldots, n$. Em cada um destes sítios existe uma comunidade de $k$ espécies que chamamos de população local ou subpopulações. Os sítios ou "patches"são fragmentos de habitat onde estão distribuídas as populações locais. Estes sítios ("patches") possuem recursos necessários para a reprodução e sobrevivência das espécies, e estão cercados por um ambiente hostil e inadequado para a sobrevivência e persistência da população.

Denotamos por $x_{k j}$ a densidade populacional da espécie $k$ no sítio $j$. Seja $\mathbf{x}_{j}=$ $\left(x_{1 j}, x_{2 j}, \ldots, x_{k j}\right) \in \mathbb{R}^{k}$ a densidade populacional das $k$ espécies no sítio $j$.

Assumimos que a dinâmica local é da forma

$$
\mathbf{x}_{j}^{t+1}=\mathbf{f}\left(\mathbf{x}_{j}^{t}\right), \quad \forall j=1,2, \ldots, n,
$$

com o operador $\mathbf{f}$ de classe $C^{1}$ dado por

$$
\begin{aligned}
& \mathbf{f}: \mathbb{R}^{k} \rightarrow \mathbb{R}^{k}, \\
&\left(x_{1 j}, x_{2 j}, \ldots, x_{k j}\right) \mapsto\left(f_{1}\left(\mathbf{x}_{j}\right), f_{2}\left(\mathbf{x}_{j}\right), \ldots, f_{k}\left(\mathbf{x}_{j}\right)\right),
\end{aligned}
$$

onde $f_{i}: \mathbb{R}^{k} \rightarrow \mathbb{R}$ para cada $i=1,2, \ldots, k$ e incorpora os processos de reprodução e sobrevivência da espécie $i$.

Estabelecemos agora conexões entre os sítios, ou seja, a possibilidade dos indivíduos de cada espécie migrarem para outros sítios. A topologia da rede, isto é, os sítios vizinhos para os quais indivíduos de um dado sítio devem migrar será definida a posteriori. Definimos abaixo o operador de dispersão (migração)

$$
\begin{aligned}
D & : \mathbb{R}^{k} \rightarrow \mathbb{R}^{k}, \\
\left(x_{1 j}, x_{2 j}, \ldots, x_{k j}\right) & \mapsto\left(D_{1}\left(\mathbf{x}_{j}\right), D_{2}\left(\mathbf{x}_{j}\right), \ldots, D_{k}\left(\mathbf{x}_{j}\right)\right),
\end{aligned}
$$


com $D_{\ell}$ dado por

$$
D_{\ell}\left(\mathbf{x}_{j}\right)=\left(I-M\left(\mathbf{x}_{j}\right)\right) \mathbf{x}_{j}+\sum_{i=1}^{n} c_{j i} M\left(\mathbf{x}_{i}\right) \mathbf{x}_{i}, \quad j=1, \ldots, n, \ell=1, \ldots, k .
$$

onde $M\left(\mathbf{x}_{j}\right)=\operatorname{diag}\left(\mu_{1}\left(\mathbf{x}_{j}\right), \mu_{2}\left(\mathbf{x}_{j}\right), \ldots, \mu_{k}\left(\mathbf{x}_{j}\right)\right), \mu_{i}: \mathbb{R}^{k} \rightarrow \mathbb{R}$ representa a fração de migração da espécie $i$ e $c_{j i}$ é a quantidade (proporção) de indivíduos que migra do sítio $i$ para o sítio $j$ (dos indivíduos que saem do sítio $i$ a proporção que migra para o sítio $j$ ). Segue que $0 \leq c_{j i} \leq 1, \forall j, i=1,2, \ldots, n$ e $c_{i i}=0$. Claramente, considerando todos os indivíduos que migram a partir do sítio $i$, temos que $\sum_{j=1}^{n} c_{j i}=1$, $\forall i=1,2, \ldots, n$, (conservação).

Supomos também que a cada geração os indivíduos passam por dois processos distintos: reprodução e sobrevivência (dinâmica local) e processo de migração. Consideramos a dinâmica local precedendo o processo de migração, ou seja, em cada geração, após o processo de dinâmica local, uma fração $\mu_{i}$ de indivíduos da espécie $i$ deixa um dado sítio e migra para os sítios mais próximos.

Segue portanto, que a dinâmica da metapopulação é dada por

$$
\mathbf{x}_{j}^{t+1}=\mathbf{f}\left(\mathbf{x}_{j}^{t}\right)-M\left(\mathbf{f}\left(\mathbf{x}_{j}^{t}\right)\right) \mathbf{f}\left(\mathbf{x}_{j}^{t}\right)+\sum_{i=1}^{n} c_{j i} M\left(\mathbf{f}\left(\mathbf{x}_{i}^{t}\right)\right) \mathbf{f}\left(\mathbf{x}_{i}^{t}\right)
$$

para todo $j=1,2, \ldots, n$. Além disso, assumimos que

$$
\sum_{i=1}^{n} c_{j i}=1, \forall j=1,2, \ldots, n .
$$

Essa é uma condição suficiente para a existência de soluções sincronizadas do sistema (2.2) (ver Seção 3).

Podemos reescrever a dinâmica da metapopulação na forma

$$
\mathbf{x}_{j}^{t+1}=\mathbf{f}\left(\mathbf{x}_{j}^{t}\right)-\sum_{i=1}^{n} b_{j i} M\left(\mathbf{f}\left(\mathbf{x}_{i}^{t}\right)\right) \mathbf{f}\left(\mathbf{x}_{i}^{t}\right)
$$

onde a matriz $B=I-C$. Observamos que $\sum_{i=1}^{n} b_{j i}=\sum_{j=1}^{n} b_{j i}=0$.

Definimos agora $\Phi: \mathbb{R}^{k} \rightarrow \mathbb{R}^{k}$ como $\Phi\left(\mathbf{x}_{\mathbf{i}}\right)=M\left(\mathbf{x}_{\mathbf{i}}\right) \mathbf{x}_{\mathbf{i}}$, e portanto a dinâmica da metapopulação é dada por

$$
\mathbf{x}_{j}^{t+1}=\mathbf{f}\left(\mathbf{x}_{j}^{t}\right)-\sum_{i=1}^{n} b_{j i} \Phi\left(\mathbf{f}\left(\mathbf{x}_{i}^{t}\right)\right), \forall j=1,2, \ldots, n .
$$

\section{Estado Sincronizado e Linearização}

Uma órbita referente ao sistema (2.2) é dita estar em estado sincronizado se, para cada $t=0,1,2, \ldots, \mathbf{x}_{i}^{t}=\mathbf{x}_{j}^{t} \equiv \mathbf{x}_{t}^{s}, \quad \forall i, j=1,2, \ldots, n$. Isso significa que o sistema está em estado sincronizado se todas as subpopulações possuem o mesmo número de indivíduos, porém a densidade populacional não se mantém necessariamente 
constante ao longo da evolução do tempo. As soluções sincronizadas assumem valores no subespaço $k$ dimensional $S=\operatorname{span}\left\{\mathbf{v}_{1}, \mathbf{v}_{2}, \ldots, \mathbf{v}_{k}\right\}$ do espaço de fase do sistema (ver Jansen \& Lloyd [15], p. 235), onde

$$
\begin{aligned}
& \mathbf{v}_{1}=\left((1,0, \ldots, 0)_{1 \times k},(1,0, \ldots, 0)_{1 \times k}, \ldots,(1,0, \ldots, 0)_{1 \times k}\right)_{n \times k}, \\
& \mathbf{v}_{2}=\left((0,1,0, \ldots, 0)_{1 \times k},(0,1,0, \ldots, 0)_{1 \times k}, \ldots,(0,1,0, \ldots, 0)_{1 \times k}\right)_{n \times k}, \\
& \vdots \\
& \mathbf{v}_{k}=\left((0, \ldots, 0,1)_{1 \times k},(0, \ldots, 0,1)_{1 \times k}, \ldots,(0, \ldots, 0,1)_{1 \times k}\right)_{n \times k} .
\end{aligned}
$$

A condição (2.3) é suficiente para que o subespaço $S$ seja invariante com relação ao sistema (2.2), e assim garante a existência de soluções sincronizadas; em particular, de pontos de equilíbrio homogêneos (ver [23]).

Seja $X_{t}^{s}=\left(\mathbf{x}_{t}^{s}, \mathbf{x}_{t}^{s}, \ldots, \mathbf{x}_{t}^{s}\right) \in \mathbb{R}^{k \times n}$ o estado sincronizado do sistema, onde $\mathbf{x}_{t}^{s}=$ $\left(x_{1}^{t}, x_{2}^{t}, \ldots, x_{k}^{t}\right)$ é o estado sincronizado da dinâmica local.

No teorema a seguir apresentamos a equação linearizada da perturbação do sistema (2.5) em torno de $X_{t}^{s}$,

Teorema 3.1. Seja $\mathbf{f}: \mathbb{R}^{k} \rightarrow \mathbb{R}^{k}$ função de classe $C^{1}$. Seja $B$ matriz $n \times n$ diagonalizável e $X_{t}^{s}=\left(\mathbf{x}_{t}^{s}, \mathbf{x}_{t}^{s}, \ldots, \mathbf{x}_{t}^{s}\right)$ o estado sincronizado da metapopulação (2.5), então o sistema linear associado a (2.5) pode ser dado na forma

$$
Y_{t+1}=\bigoplus_{j=0}^{n-1}\left[I-\lambda_{j} D \Phi\left(\mathbf{f}\left(\mathbf{x}_{t}^{s}\right)\right)\right] D \mathbf{f}\left(\mathbf{x}_{t}^{s}\right) Y_{t}
$$

onde $Y_{t}=\left(\mathbf{y}_{1}, \mathbf{y}_{2}, \ldots, \mathbf{y}_{k}\right) \in \mathbb{R}^{k \times n}$ e $\mathbf{y}_{j}=\left(y_{1 j}, y_{2 j}, \ldots, y_{k j}\right) \in \mathbb{R}^{k}$. Além disso, $\lambda_{j}$ são os autovalores da matriz $B=I-C$.

Demonstração. Linearizando o sistema (2.5) em torno do estado sincronizado $X_{t}^{s}$, obtemos a seguinte equação para a evolução da perturbação, $\Delta_{t}$,

$$
\Delta_{t+1}=J\left(X_{t}^{s}\right) \Delta_{t}
$$

onde $X_{t}=X_{t}^{s}+\Delta_{t}$ e $J\left(X_{t}^{s}\right)$ é a matriz jacobiana $(n k) \times(n k)$ do sistema (2.5) dada por $J\left(X_{t}^{s}\right)=I \otimes D \mathbf{f}\left(\mathbf{x}_{t}^{s}\right)-B \otimes D \Phi\left(\mathbf{f}\left(\mathbf{x}_{t}^{s}\right)\right) D \mathbf{f}\left(\mathbf{x}_{t}^{s}\right)$, e $\otimes$ representa o produto de Kronecker ${ }^{3}$.

Por hipótese $B$ é diagonalizável, então existe $P$ matriz não singular que diagonaliza $B$, isto é, $P B P^{-1}=\Lambda$.

Consideremos a seguinte mudança de variáveis $Y_{t}=(P \otimes I) \Delta_{t}$, assim segue-se que

$$
\begin{aligned}
Y_{t+1} & =(P \otimes I) \Delta_{t+1} \\
& =(P \otimes I)\left[I \otimes D \mathbf{f}\left(\mathbf{x}_{t}^{s}\right)-B \otimes D \Phi\left(\mathbf{f}\left(\mathbf{x}_{t}^{s}\right)\right) D \mathbf{f}\left(\mathbf{x}_{t}^{s}\right)\right] \Delta_{t} \\
& =\left[\left(P \otimes D \mathbf{f}\left(\mathbf{x}_{t}^{s}\right)\right)-P B \otimes D \Phi\left(\mathbf{f}\left(\mathbf{x}_{t}^{s}\right)\right) D \mathbf{f}\left(\mathbf{x}_{t}^{s}\right)\right] \Delta_{t},
\end{aligned}
$$

\footnotetext{
${ }^{3}$ Seja $A=\left[a_{i j}\right]_{i, j=1}^{m} \in \mathbb{R}^{m \times m}$ e $B=\left[b_{i j}\right]_{i, j=1}^{n} \in \mathbb{R}^{n \times n}$, o produto de Kronecker é definido por $A \otimes B=\left[a_{i j} B\right]_{i, j=1}^{m} \in \mathbb{R}^{m n \times m n}$.
} 
pela propriedade do produto de Kronecker (ver Proposição 2 p. 408 [17]).

Como $\Delta_{t}=(P \otimes I)^{-1} Y_{t}$, e utilizando as propriedades do produto de Kronecker temos

$$
\begin{aligned}
Y_{t+1} & =\left[\left(P \otimes D \mathbf{f}\left(\mathbf{x}_{t}^{s}\right)\right)-P B \otimes D \Phi\left(\mathbf{f}\left(\mathbf{x}_{t}^{s}\right)\right) D \mathbf{f}\left(\mathbf{x}_{t}^{s}\right)\right](P \otimes I)^{-1} Y_{t} \\
& =\left[\left(P \otimes D \mathbf{f}\left(\mathbf{x}_{t}^{s}\right)\right)-P B \otimes D \Phi\left(\mathbf{f}\left(\mathbf{x}_{t}^{s}\right)\right) D \mathbf{f}\left(\mathbf{x}_{t}^{s}\right)\right]\left(P^{-1} \otimes I\right) Y_{t} \\
& =\left[I \otimes D \mathbf{f}\left(\mathbf{x}_{t}^{s}\right)-\Lambda \otimes D \Phi\left(\mathbf{f}\left(\mathbf{x}_{t}^{s}\right)\right) D \mathbf{f}\left(\mathbf{x}_{t}^{s}\right)\right] Y_{t} .
\end{aligned}
$$

Portanto, como $\Lambda=\operatorname{diag}\left(\lambda_{0}, \lambda_{1}, \ldots, \lambda_{n-1}\right)$, onde os $\lambda_{i}$ são os autovalores de $B$, temos o resultado desejado.

\section{Exemplo - Dinâmica Local com Hierarquia}

Neste trabalho, consideramos a dinâmica local com hierarquia. Cada espécie depende somente daqueles que estão acima do seu nível, ou seja, $f_{1}$ depende da espécie $1, f_{2}$ depende das espécies 1 e 2 e assim sucessivamente de tal forma que cada $f_{i}$ depende de $i$ espécies. A forma hierárquica pode ser caracterizada por vários fatores, entre eles o clima, quantidade de recursos para a sobrevivência, habilidades competitivas (primeira espécie é a melhor competidora, enquanto que a última espécie envolvida apresenta o pior comportamento competitivo). Este comportamento hierárquico está presente na natureza em espécies de cracas (crustáceos), onde os indivíduos menos fortes são eliminados.

Dessa forma a matriz jacobiana $D \mathbf{f}$ associada ao modelo desacoplado proposto é dada por uma matriz triangular inferior $k \times k$

$$
D \mathbf{f}=\left(\begin{array}{ccccc}
\frac{\partial f_{1}}{\partial x_{1}} & 0 & \ldots & \ldots & 0 \\
\frac{\partial f_{2}}{\partial x_{1}} & \frac{\partial f_{2}}{\partial x_{2}} & 0 & \ldots & 0 \\
\vdots & \vdots & \ddots & \ddots & \vdots \\
\vdots & \vdots & & \ddots & 0 \\
\frac{\partial f_{k}}{\partial x_{1}} & \frac{\partial f_{k}}{\partial x_{2}} & \ldots & \ldots & \frac{\partial f_{k}}{\partial x_{k}}
\end{array}\right)
$$

Modelos desta natureza foram investigados por Best et al. [2] para competição. A evolução do sistema com hierarquia apresentado por eles é dada por

$$
x_{k}(t+1)=x_{k}(t) g_{k}\left(\sum_{j=1}^{k} \alpha_{k j} x_{j}(t)\right), k=1,2, \ldots, n
$$

onde $x_{k}(t)$ representa a densidade populacional da espécie $k$ na geração $t, \alpha_{k j}$ mede a taxa do efeito competitivo da espécie $k$ para a espécie $j$ e $g_{i}:[0,+\infty) \rightarrow[0,+\infty)$ função estritamente crescente, positiva e diferenciável que representa o crescimento da espécie $i$. 


\section{Estabilidade do Estado Sincronizado}

Nesta seção analisamos a estabilidade do estado sincronizado do sistema (2.5). Para isso consideramos o sistema linearizado (ver Teorema 3.1) de (2.5)

$$
Y_{t+1}=\bigoplus_{j=0}^{n-1}\left[I-\lambda_{j} D \Phi\left(\mathbf{f}\left(\mathbf{x}_{t}^{s}\right)\right)\right] D \mathbf{f}\left(\mathbf{x}_{t}^{s}\right) Y_{t}
$$

Supomos que cada fração de migração $\mu_{i}$ depende apenas da densidade da espécie $i$. Assim, a matriz $D \Phi$ é uma matriz diagonal $k \times k$ da forma

$$
D \Phi=\operatorname{diag}\left(\frac{\partial \mu_{1}}{\partial x_{1}} f_{1}+\mu_{1}, \ldots, \frac{\partial \mu_{k}}{\partial x_{k}} f_{k}+\mu_{k}\right) .
$$

Seja $T=\bigoplus_{j=0}^{n-1}\left[I-\lambda_{j} D \Phi\left(\mathbf{f}\left(\mathbf{x}_{t}^{s}\right)\right)\right] D \mathbf{f}\left(\mathbf{x}_{t}^{s}\right)$ a matriz diagonal por blocos associada ao sistema (3.1). Cada $j$-bloco $\left[I-\lambda_{j} D \Phi\left(\mathbf{f}\left(\mathbf{x}_{t}^{s}\right)\right)\right] D \mathbf{f}\left(\mathbf{x}_{t}^{s}\right)$ será denominado por $T_{j}$. Por hipótese $D \Phi$ é diagonal. Logo, $I-\lambda_{j} D \Phi$ é diagonal. O produto das matrizes $I-\lambda_{j} D \Phi$ (diagonal) por $D \mathbf{f}$ (triangular inferior) é uma matriz triangular inferior ${ }^{4}$. Portanto, cada $j$-bloco é uma matriz triangular inferior.

A órbita caótica sincronizada vive ao longo do subespaço $S$ do espaço de fase que é invariante sobre a dinâmica. Como $\sum b_{i j}=0$, então $\lambda_{0}=0$ é autovalor de $B$ associado ao autovetor $(1,1, \ldots, 1)^{T}$. Logo, o bloco $T_{0}$ está exatamente sobre o subespaço $S$ invariante. Portanto, o critério para estabilidade local do estado sincronizado do sistema (2.2), será obtido investigando apenas as perturbações transversais ao sistema, e está descrito no teorema a seguir.

Teorema 5.1. Sejam $C$ uma matriz $n \times n$ duplamente estocástica $\operatorname{com} c_{i i}=0, i=$ $1,2, \ldots, n, \mathbf{f}: \mathbb{R}^{k} \rightarrow \mathbb{R}^{k}$ uma função de classe $C^{1}, \lambda_{j}$ para $j=0, \ldots, n-1$ os autovalores da matriz $B=I-C$, e $\rho$ a medida (ergódica) de probabilidade natural do sistema local (2.1). Então os $(n-1) k$ números transversais de Liapunov são dados pela expressão

$$
\widetilde{\Lambda}_{i}^{j}=\exp \int_{\mathbb{R}^{k}} \ln \left|1-\lambda_{j} \frac{\partial \phi_{i}}{\partial x_{i}}(\mathbf{x})\right|\left|\frac{\partial f_{i}}{\partial x_{i}}(\mathbf{x})\right| d \rho(\mathbf{x}),
$$

e o atrator do sistema (3.1) no conjunto sincronizado invariante é estável se

$$
\Lambda<1
$$

onde $\Lambda=\max _{i=1: k ; j=1: n-1} \widetilde{\Lambda}_{i}^{j}$.

Demonstração. Os expoentes de Liapunov são definidos como os autovalores da matriz $\Lambda_{\mathbf{x}}=\lim _{\tau \rightarrow \infty}\left(T_{\mathbf{x}}^{\tau *} T_{\mathbf{x}}^{\tau}\right)^{\frac{1}{2 \tau}}$, onde

$$
T^{\tau}=\prod_{t=0}^{\tau-1} \bigoplus_{j=1}^{n-1}\left[I-\lambda_{j} D \Phi\left(\mathbf{f}\left(\mathbf{x}_{t}^{s}\right)\right)\right] D \mathbf{f}\left(\mathbf{x}_{t}^{s}\right) .
$$

\footnotetext{
${ }^{4}$ Seja $D$ matriz diagonal com entradas $d_{i}, i=1, \ldots, n$ e $A$ matriz triangular inferior com entradas $a_{i j}\left(a_{i j}=0\right.$ se $\left.j>i\right)$, então a matriz produto $P=D A$ com entradas $p_{i j}$ é tal que $p_{i j}=0$ se $j>i$ e $p_{i j}=d_{i} a_{i j}$ para $j \leq i$.
} 
A existência desse limite é garantida pelo Teorema Ergódico Multiplicativo de Oseledec (ver [10]).

A matriz $T^{\tau}$ é diagonal por blocos. Cada $j$-bloco é dado por $T_{j}^{\tau}=\left[I-\lambda_{j} D \Phi\left(\mathbf{x}_{0}^{s}\right)\right]$ $D \mathbf{f}\left(\mathbf{x}_{0}^{s}\right) \cdots\left[I-\lambda_{j} D \Phi\left(\mathbf{x}_{\tau-1}^{s}\right)\right] D \mathbf{f}\left(\mathbf{x}_{\tau-1}^{s}\right)$. A matriz $\left[I-\lambda_{j} D \Phi(\mathbf{x})\right] D \mathbf{f}(\mathbf{x})$ é triangular inferior (ver início da seção 5.). Do fato que o produto de matriz triangular inferior resulta uma matriz triangular inferior segue que o $j$-ésimo bloco $T_{j}^{\tau}$ de $T^{\tau}$ é uma matriz triangular inferior com elementos da diagonal dados por $z_{i i}=\left(1-\lambda_{j} \frac{\partial \phi_{i}}{\partial x_{i}}\left(\mathbf{x}_{0}^{s}\right)\right) \frac{\partial f_{i}}{\partial x_{i}}\left(\mathbf{x}_{0}^{s}\right) \cdots\left(1-\lambda_{j} \frac{\partial \phi_{i}}{\partial x_{i}}\left(\mathbf{x}_{\tau-1}^{s}\right)\right) \frac{\partial f_{i}}{\partial x_{i}}\left(\mathbf{x}_{\tau-1}^{s}\right)$.

Como o sistema (3.1) no estado sincronizado é regular (ver [21] p. 465), e a matriz $T$ é uma matriz triangular, segue que os expoentes transversais de Liapunov do sistema são dados por (ver [6])

$$
\Lambda_{i}^{j}=\lim _{\tau \rightarrow \infty} \frac{1}{\tau} \sum_{t=0}^{\tau-1} \ln \left|\left(1-\lambda_{j} \frac{\partial \phi_{i}}{\partial x_{i}}\left(\mathbf{x}_{t}^{s}\right)\right) \frac{\partial f_{i}}{\partial x_{i}}\left(\mathbf{x}_{t}^{s}\right)\right|,
$$

para $i=1, \cdots, k, j=1, \cdots, n-1$. Logo, pelo teorema ergódico de Birkoff (ver [28] p. 35 e [10]) tem-se que os expoentes transversais de Liapunov são da forma

$$
\Lambda_{i}^{j}=\int_{\mathbb{R}^{k}} \ln \left|1-\lambda_{j} \frac{\partial \phi_{i}}{\partial x_{i}}(\mathbf{x})\right|\left|\frac{\partial f_{i}}{\partial x_{i}}(\mathbf{x})\right| d \rho(\mathbf{x}),
$$

$i=1, \cdots, k, j=1, \cdots, n-1$.

Definimos

$$
\widetilde{\Lambda}_{i}^{j}=\exp \int_{\mathbb{R}^{k}} \ln \left|1-\lambda_{j} \frac{\partial \phi_{i}}{\partial x_{i}}(\mathbf{x})\right|\left|\frac{\partial f_{i}}{\partial x_{i}}(\mathbf{x})\right| d \rho(\mathbf{x}),
$$

$i=1, \cdots, k, j=1, \cdots, n-1$ os números de Liapunov transversais associados ao sistema $Y_{t+1}=T Y_{t}$.

Consideramos agora o máximo dos números de Liapunov de cada bloco,

$$
\widetilde{\Lambda}^{1}=\max _{i=1, \cdots, k} \widetilde{\Lambda}_{i}^{1}, \quad \widetilde{\Lambda}^{2}=\max _{i=1, \cdots, k} \widetilde{\Lambda}_{i}^{2}, \cdots, \quad \widetilde{\Lambda}^{n-1}=\max _{i=1, \cdots, k} \widetilde{\Lambda}_{i}^{n-1} .
$$

e, finalmente o máximo desses, isto é, $\Lambda=\max _{j=1, \cdots, n-1} \widetilde{\Lambda}^{j}$.

Portanto, o critério de estabilidade do estado sincronizado é $\Lambda<1$.

\section{Conclusão}

O fenômeno de sincronização em modelos biológicos acoplados tem recebido crescente interesse na literatura (ver [7], [24], [25]). Estudamos um modelo metapopulacional de $k$ espécies com migração dependente da densidade estendendo os resultados de [15].

Vários exemplos podem ser considerados para a dinâmica local do sistema constituída de $k$ espécies. Estas $k$ espécies podem ser abordadas como $k$ espécies distintas interagindo no sistema, é o caso do modelo geral para a interação hospedeiroparasitóide considerado por Rohani et al. [20]

$$
\begin{aligned}
H_{t+1} & =\lambda H_{t} F\left(P_{t}\right) \\
P_{t+1} & =c H_{t}\left(1-F\left(P_{t}\right)\right),
\end{aligned}
$$


onde $H_{t}$ é a densidade dos hospedeiros no tempo $t, P_{t}$ a densidade dos parasitóides no tempo $t, \lambda$ a taxa de crescimento dos hospedeiros, $F\left(P_{t}\right)$ probabilidade do hospedeiro escapar do parasitismo e $c$ número de parasitóides que emergem a partir de um hospedeiro parasitado. Outra forma de considerarmos $k$ espécies no sistema são os modelos estruturados por classes etárias. Cada classe etária representa uma espécie. Neste caso, em cada sítio temos uma única espécie com suas $k$ classes etárias. A dinâmica local para estes casos pode ser dada por $\mathbf{x}_{t+1}=F\left(\mathbf{x}_{t}\right)=$ $\left(\begin{array}{ccccc}f_{1} & f_{2} & \cdots & f_{k-1} & f_{k} \\ p_{1} & 0 & \cdots & & 0 \\ 0 & p_{2} & 0 & \cdots & 0 \\ 0 & \cdots & \ddots & & \\ 0 & & & p_{k-1} & 0\end{array}\right) \mathbf{x}_{t}$, onde $\mathbf{x}_{t}=\left(x_{1}^{t}, \ldots, x_{k}^{t}\right), x_{k}^{t}$ população na classe etária $k$ no tempo $t$ e $f_{i}$ taxa de fertilidade da classe etária $i, p_{i}$ probabilidade de sobrevivência. Estes modelos são investigados desde os trabalhos pioneiros de Leslie. Em Levin \& Goodyear [18], Silva \& Hallam [26] e Wikan \& Mjølhus [29] algumas questões fundamentais como estabilidade, bifurcações e oscilações para estes modelos com estrutura etária são discutidos. De Castro et al. [5] consideraram modelos com classes etárias, um modelo linear baseado no modelo clássico de Leslie e outro não linear adicionando recrutamento dependente da densidade. Em ambos os casos condições para a estabilidade da dinâmica local e global foram obtidas. Os modelos epidemiológicos, SIS, SIR, SEIR (ver [16] e [3]) onde, em cada sítio, existem vários estágios da doença para uma determinada população de única espécie, também podem ser considerados para esta forma de dinâmica local. Castillo-Chavez e Yakubu [3]) analisaram o modelo S-I-S

$$
\begin{aligned}
S_{t+1} & =f\left(T_{t}\right)+\gamma S_{t} G\left(\alpha \frac{I_{t}}{T_{t}}\right)+\gamma T_{t}(1-\sigma) \\
I_{t+1} & =\gamma\left[1-G\left(\alpha \frac{I_{t}}{T_{t}}\right)\right] S_{t}+\gamma \sigma I_{t},
\end{aligned}
$$

onde $S_{t}$ indivíduos suscetíveis, $I_{t}$ indivíduos infectados, e $T_{t}=S_{t}+I_{t}$ população total. Além disso, $\gamma$ é a probabilidade de sobrevivência dos indivíduos em cada geração $(1-\gamma$ probabilidade de morte), $1-\sigma$ probabilidade de recuperação de indivíduos infectados ( $\sigma$ não se recuperam), $1-G$ probabilidade de suscetíveis tornarem-se infectados ( $G$ permanecem suscetíveis).

Neste trabalho a dinâmica local considerada foi a hierárquica. Para este caso, obtivemos uma expressão analítica para os números transversais de Liapunov associados ao sistema e, como conseqüência, um critério para a estabilidade local do estado sincronizado.

\footnotetext{
Abstract. In this paper we analyze the possibility of the existence of the synchronized oscillation of chaotic orbits for metapopulations models of $k$ species under density-dependence migration. We consider the local dynamics in the hierarchical form and obtain a criterion for the stability of synchronized state of the chaotic synchronized orbits.
} 


\section{Referências}

[1] J.C. Allen, W.M. Schaffer, D. Rosko, Chaos reduces species extinction by amplifying local population noise, Nature, 364 (1993), 229-232.

[2] J. Best, C. Castillo-Chavez, A-A. Yakubu, Hierarchical Competition in Discrete Time Models with Dispersal, Fields Institutional of Communications, 36 (2003), 59-86.

[3] C. Castillo-Chavez, A-A. Yakubu, Discrete time S-I-S models with complex dynamics, Nonlinear Analysis, 47 (2001), 4753-4762.

[4] H. N. Comins, The spatial dynamics of host-parasitoid systems, J. Anim. Ecol., 61 (1992), 735-748.

[5] M.L. de Castro, J.A.L. Silva, D.A.R. Justo, Stability in an-structured metapopulation model, J. Math. Biology, 52 (2006) 183-208.

[6] L. Dieci, E.S. Van Vleck, Computation of a few Lyapunov exponents for continuous and discrete dynamical systems, Appl. Numer. Math., 17 (1995), 275291.

[7] M. Ding, W. Yang, Stability of synchronous and on-off intermittency in coupled map lattices, Physical Review E, 56 (1997), 4009-4016.

[8] A.S. Dmitriev, M. Shirokov, S.O. Starkov, Chaotic synchronization in ensembles of coupled maps, IEEE Transactions on Circuits and Systems- : Fundamental Theory and Applications, 44 (1997), 918-926.

[9] D.J.D. Earn, S.A. Levin, P. Rohani, Coherence and conservation, Science, 290 (2000), 1360-1364.

[10] J.P. Eckmann, D. Ruelle, Ergodic theory of chaos and strange attractors, Am. Physical Society, 57 (1985), 617-656.

[11] A. Gonzalez, J. H. Lawton, F. S. Gilbert, T. M. Blackburn, I. Evans-Freke, Metapopulation dynamics, abundance, and distribution in a microsystem, Science, 281 (1998), 2045-2047.

[12] M.P. Hassell, H.N. Comins, R.M. May, Spatial structure and chaos in insect population dynamics, Nature, 353 (1991), 255-258.

[13] M. Heino, V. Kaitala, E. Ranta, J. Lindström, Synchonous dynamics and rates of extinction in spatially structured populations, Proc. Royal Soc. London B, 264 (1997), 481-486.

[14] R.A. Ims, H.P. Andreassen, Density-dependent dispersal and spatial population dynamics, Proc. Roy. Soc. B, 272 (2005) 913-918.

[15] V.A.A. Jansen, A.L. Lloyd, Local stability analysis of spatially homogeneous solutions of multi-patch systems, J. Math. Biol., 41 (2000), 232-252. 
[16] A.L. Lloyd, V.A.A. Jansen, Spatiotemporal dynamics of epidemics: synchrony in metapopulation models, Math. Biosc., 188 (2004), 1-16.

[17] P. Lancaster, M. Tismenetsky, "The Theory of Matrices", Academic Press, London, 1985.

[18] S.A. Levin, C.P. Goodyear, Analysis of an age-structured fishery model, $J$. Math. Biol., 9 (1980), 245-274.

[19] A. Pikovsky, M. Rosenblum, J. Kurths, "Synchronization: a universal concept in nonlinear sciences", Cambridge University Press, Cambridge, 2001.

[20] P. Rohani, R.M. May, M.P. Hassell, Metapopulation and equilibrium stability: the effects of spatial structure, J. Theor. Biol. , 181 (1996), 97-109.

[21] G. Sansone, R. Conti, "Non-Linear Differential Equations", The Macmillan Company, New York, 1964.

[22] J.A.L. Silva, J.A. Barrionuevo, F.T. Giordani, Synchronism in populations networks with non linear coupling, submetido, 2006.

[23] J.A.L. Silva, M.L.D. Castro, D.A.R. Justo, Stability in a metapopulation model with density-dependent dispersal, Bull. Math. Biol., 63 (2001), 485-506.

[24] J.A.L. Silva, M.L.D. Castro, D. A. R. Justo, Synchronism in a metapopulation model, Bull. Math. Biol., 62 (2000), 337-349.

[25] J.A.L. Silva, F.T. Giordani, Density-dependent migration and synchronism in metapopulations, Bull. Math. Biol., 68 (2006), 451-465.

[26] J.A.L. Silva, T.G. Hallam, Compensation and stability in nonlinear matrix models, Math. Bios., 31 (1992), 67-101.

[27] R.V. Solé, J.P.G. Gamarra, Chaos, dispersal and extinction in coupled ecosystems, J. Theor. Biol., 193 (1998), 539-541.

[28] P. Walters, "An Introduction to Ergodic Theory", Springer, New York, 1982.

[29] A. Wikan, E. Mjølhus, Periodicity of 4 in Age-structured population models with density dependence, J. Theor. Biol., 173 (1995), 109-119. 